\title{
Clinical Lung Cancer
}

Brown-Séquard syndrome in a desmoplastic malignant mesothelioma patient: A rare complication

--Manuscript Draft--

\begin{tabular}{|l|l|}
\hline Manuscript Number: & CLC-D-20-00638R1 \\
\hline Article Type: & Case Report \\
\hline Keywords: & Brown-Séquard syndrome; Malignant mesothelioma; Spinal metastasis \\
\hline Corresponding Author: & $\begin{array}{l}\text { Mike Ralki, MD } \\
\text { Katholieke Universiteit Leuven Universitaire Ziekenhuizen Leuven } \\
\text { BELGIUM }\end{array}$ \\
\hline First Author: & Mike Ralki, MD \\
\hline Order of Authors: & Mike Ralki, MD \\
\hline \multirow{5}{*}{ Anouk Serrien, MD } \\
\hline & Julie Lambert, MD \\
\hline & Mathias Leys, MD \\
\hline & Kristiaan Nackaerts, MD, PhD \\
\hline
\end{tabular}


Reviewers

Clinical Lung Cancer

September 9, 2020

Dear Reviewers:

We greatly appreciate your comments to our manuscript and we have revised the manuscript accordingly. In the following, we give a point-by-point reply to your comments:

Reviewer 1: Did the patient improve at all after radiation? Practicing clinicians may want to know. Desmoplastic cancers of all types, including mesothelioma, are not as responsive to this modality. Were steroids administered and did they help as well?

We agree that desmoplastic mesothelioma cells are only modestly radiosensitive. Our patient was treated with radiation therapy (25Gy on the primary MM tumor and 20Gy on the spinal metastasis) instead of second-line systemic therapy because of the rather localized aspect of malignant disease and to achieve additional pain control in his right shoulder/neck. Steroids (methylprednisolone 16mg/day for 1 week and methylprednisolone $8 \mathrm{mg} /$ day for 1 week) were added during the radiation therapy as an adjunctive anti-inflammatory treatment. There was no significant clinical improvement after this therapy and repeat CT one month later showed stable disease. In the months thereafter, his neurological status deteriorated and he received best supportive care.

Reviewer 2: Dr. Ralki and colleagues present an interesting case of desmoplastic mesothelioma with spine metastases causing neurologic symptoms. While the ability of mesothelioma to metastasize distantly is well recognized, it is not reflected well in the literature and this case report thoughtfully adds to that literature. My only comment would be to more specifically discuss what has been reported about this for mesothelioma, albeit not desmoplastic, in the introduction.

We fully agree that the ability of mesothelioma to spread hematogenously is underestimated both in the literature and in clinical practice. Postmortem studies are sparse, the largest and most recent postmortem study $(n=318)$ found extrathoracic metastases in $55.4 \%$ and central nervous metastases in $3 \%$ of mesothelioma patients (1). In living patients, only one retrospective cohort study ( $n=165)$ addressed this issue. Distant metastases were reported in up to $27 \%$ of all mesothelioma patients, depending on the affected organ site (2). One report $(n=27)$ suggests that desmoplastic mesothelioma tend to metastasize more frequently than non-desmoplastic mesothelioma (3).

For your convenience, all changes to the original manuscript and additional references are clearly marked in red in the revised manuscript. In addition, we have added a second copy of the revised manuscript without any visible indications.

Yours sincerely

Mike Ralki, Anouk Serrien, Julie Lambert, Mathias Leys and Kristiaan Nackaerts 


\section{References:}

1. Finn R, Brims F, Gandhi A, Olsen N, Musk A, Maskell N et al. Postmortem findings of malignant pleural mesothelioma: a two-center study of 318 patients. Chest 2012;142(5):12671273.

2. Collins DC, Constantinidou A, Sundar R, Chenard-Poirier M, Yap TA, Banerji U et al. Patterns of metastases in malignant pleural mesothelioma in the modern era: Redefining the spread of an old disease. J Clin Oncol 2017;35(15):8556.

3. Cantin R, Jabi MA, McCaughey WT. Desmoplastic diffuse mesothelioma. Am J Surg Pathol 1982;6(3):215-222. 


\title{
Brown-Séquard syndrome in a desmoplastic malignant mesothelioma patient: A rare complication
}

\author{
Mike Ralki, $\mathrm{MD}^{1 *}$ \\ Anouk Serrien, $\mathrm{MD}^{2 *}$ \\ Julie Lambert, $\mathrm{MD}^{3}$ \\ Mathias Leys, $\mathrm{MD}^{4}$ \\ Kristiaan Nackaerts, $\mathrm{MD}, \mathrm{PhD}^{1}$ \\ ${ }^{1}$ Department of Pneumology, KU Leuven University Hospitals Leuven, Leuven, Belgium \\ ${ }^{2}$ Department of Neurology, KU Leuven University Hospitals Leuven, Leuven, Belgium \\ ${ }^{3}$ Department of Radiology, KU Leuven University Hospitals Leuven, Leuven, Belgium \\ ${ }^{4}$ Department of Pneumology, AZ Groeninge Hospital, Kortrijk, Belgium \\ * Equally contributed
}

Corresponding author: Mike Ralki, Department of Pneumology, KU Leuven University Hospitals Leuven, Herestraat 49, B-3000 Leuven, Belgium, mike.ralki@uzleuven.be

All authors declare no conflicts of interest related to this paper. 


\section{$\underline{\text { Introduction }}$}

Desmoplastic malignant mesothelioma is a rare histological subtype of malignant mesothelioma (MM), generally bearing a poor prognosis due to aggressive local infiltration and the development of distant metastases. Hematogenous metastases in MM are considered rare, although postmortem studies show extrathoracic metastases in up to 55\% of MM patients and central nervous metastases in 3\% of MM patients (1). In living patients, only one retrospective cohort study $(n=165)$ addressed this issue. Distant metastases were reported in up to $27 \%$ of all MM patients, depending on the affected organ site (2). Desmoplastic MM tends to metastasize more frequently than non-desmoplastic MM (3).

Neurological complaints in MM are common and may be attributed to peripheral causes such as peroneal nerve palsy (secondary to weight loss) or to treatment-related side effects (amongst others, polyneuropathy secondary to chemotherapeutics and immunotherapy-related toxicity). However, neurological symptoms due to metastasis to the central nervous system in a desmoplastic mesothelioma have never been reported before to our knowledge.

We present a case report of a desmoplastic MM patient with right leg paresis caused by a spinal cord metastasis.

\section{Clinical practice points}

- The differential diagnosis of neurological complaints in patients with malignant mesothelioma is broad.

- Hematogenous spinal cord metastasis of malignant mesothelioma is rare and has not been reported before in desmoplastic malignant mesothelioma.

- This case presents a desmoplastic malignant mesothelioma patient with right leg paresis caused by a spinal cord metastasis.

- This case demonstrates that spinal cord metastasis should be considered as a cause of neurological complaints in patients with malignant mesothelioma, especially when clinical examination suggests spinal cord pathology.

\section{Case report}

A 66-year-old male patient was diagnosed with a MM localized in the apical part of his right pleural cavity and invading the brachial plexus. Three months before, he underwent a foraminotomy at level C4-C7 for pain in his right neck and shoulder, without any improvement afterwards. After his MM was considered inoperable, he was treated with the combination of carboplatin (AUC5), pemetrexed $500 \mathrm{mg} / \mathrm{m}^{2}$, bevacizumab $15 \mathrm{mg} / \mathrm{kg}$ and atezolizumab $1200 \mathrm{mg}$ (after inclusion in the Multicenter Randomized Phase III 'BEAT-meso' trial of the European Thoracic Oncology Platform) (4). Under this treatment, he achieved a best response of 'stable disease'.

Five months after MM diagnosis, he was hospitalized because of paresis of the right leg with difficulties maintaining his equilibrium. Clinically, he had Brown-Séquard syndrome: pyramidal signs in the right leg (reduced muscle power, positive Babinski sign, slightly higher muscle tone and ankle clonus) and thermoanalgesia in the left hemicorpus, approximately up to level T3. On electrodiagnostic studies, a severe polyneuropathy was discovered, explaining why his deep sensation was severely diminished in both lower extremities.

Imaging of the spinal cord did not only reveal local spread of the MM at level T1-T2, but also a rightsided spinal metastasis at level C7-T1, compatible with the clinical findings (Figure 1,2). After 
multidisciplinary discussion, the BEAT trial treatment was stopped and locoregional radiotherapy on the primary MM tumor (25Gy) and the spinal metastasis (20Gy) was initiated, in combination with systemic steroids (methylprednisolone $16 \mathrm{mg}$ /day for 1 week and methylprednisolone $8 \mathrm{mg} / \mathrm{day}$ for 1 week) as an adjunctive anti-inflammatory treatment during the course of radiotherapy. There was no significant clinical improvement after this therapy and repeat CT one month later showed stable disease. In the months thereafter, his neurological status deteriorated and he received best supportive care.

\section{$\underline{\text { Discussion }}$}

Some case series already described the rare formation of intramedullary metastases in MM; however, none of these cases was of the histological desmoplastic subtype $(5,6)$. The mechanism of MM dissemination to the spinal cord remains intriguing but unprovable with imaging in our case. In figure $3 a$, we visualize a blood vessel with pathological widening and contrast captation, which originates in the lower intramedullary foramen. Consequently, hematogenous spread from the expanding primary MM tumor to this metastasis may have occurred via this route. In addition, local spread from the MM is visualized in figure $3 b$, extending into the pleura and soft tissue of the lung, further expanding in the right intervertebral foramen at level T1-T2, but not expanding into the extramedullar intraspinal space. Possibly the foraminotomy in our patient's history, with widening of the intervertebral spaces C4-C7, contributed to the local spread. Both hematogenous and local spread have been proposed as mechanisms of action in similar cases (5-7).

\section{References}

1. Finn R, Brims F, Gandhi A, Olsen N, Musk A, Maskell N et al. Postmortem findings of malignant pleural mesothelioma: a two-center study of 318 patients. Chest 2012;142(5):12671273.

2. Collins DC, Constantinidou A, Sundar R, Chenard-Poirier M, Yap TA, Banerji U et al. Patterns of metastases in malignant pleural mesothelioma in the modern era: Redefining the spread of an old disease. J Clin Oncol 2017;35(15):8556.

3. Cantin R, Jabi MA, McCaughey WT. Desmoplastic diffuse mesothelioma. Am J Surg Pathol 1982;6(3):215-222.

4. BEAT-meso: Bevacizumab and Atezolizumab in Malignant Pleural Mesothelioma (NCT03762018). Available from:

https://clinicaltrials.gov/ct2/show/NCT03762018?term=BEAT\&cond=Mesotheliomas+Pleural $\&$ draw $=2 \&$ rank $=1$ (accessed on June 01, 2020).

5. Yamamoto J, Ueta K, Takenaka M, Takahashi M, Nishizawa S. Sarcomatoid malignant mesothelioma presenting with intramedullary spinal cord metastasis: A case report and literature review. Global Spine J 2014;4(2):115-120.

6. Kalfas F, Skudieri C. Intramedullary Metastasis to the Cervical Spinal Cord from Malignant Pleural Mesothelioma: Review of the Literature and Case Reports. Asian J Neurosurg 2020;15(1):70-75.

7. Hillard VH, Liu JK, Kwok A, Schmidt MH. Perineural Spread of Malignant Mesothelioma Resulting in an Intradural Spinal Cord Mass: Case Report. J Neurooncol 2007; 81(2):185-89. 


\section{Figure legends}

Figure 1: Right paramedian sagittal T1-weighted image, showing the intramedullary mass lesion (larger white arrow) and posterior epidural lesion (smaller white arrow).

Figure 2: Median sagittal STIR image of the intramedullary mass lesion with surrounding edema.

Figure 3: Figure 3a (upper figure): Axial T1-weighted image after contrast with visualization of a pathologically thickened blood vessel against the tumor (white arrow). Figure $3 b$ (lower figure): Axial T1-weighted image after contrast, showing the extension of the tumor from the apex of the right lung to the intervertebral foramen (between arrows). 


\title{
Brown-Séquard syndrome in a desmoplastic malignant mesothelioma patient: A rare complication
}

\author{
Mike Ralki, $\mathrm{MD}^{1 *}$ \\ Anouk Serrien, $\mathrm{MD}^{2 *}$ \\ Julie Lambert, $\mathrm{MD}^{3}$ \\ Mathias Leys, $\mathrm{MD}^{4}$ \\ Kristiaan Nackaerts, $\mathrm{MD}, \mathrm{PhD}^{1}$ \\ ${ }^{1}$ Department of Pneumology, KU Leuven University Hospitals Leuven, Leuven, Belgium \\ ${ }^{2}$ Department of Neurology, KU Leuven University Hospitals Leuven, Leuven, Belgium \\ ${ }^{3}$ Department of Radiology, KU Leuven University Hospitals Leuven, Leuven, Belgium \\ ${ }^{4}$ Department of Pneumology, AZ Groeninge Hospital, Kortrijk, Belgium \\ * Equally contributed
}

Corresponding author: Mike Ralki, Department of Pneumology, KU Leuven University Hospitals Leuven, Herestraat 49, B-3000 Leuven, Belgium, mike.ralki@uzleuven.be

All authors declare no conflicts of interest related to this paper. 


\section{$\underline{\text { Introduction }}$}

Desmoplastic malignant mesothelioma is a rare histological subtype of malignant mesothelioma (MM), generally bearing a poor prognosis due to aggressive local infiltration and the development of distant metastases. Hematogenous metastases in MM are considered rare, although postmortem studies show extrathoracic metastases in up to 55\% of MM patients and central nervous metastases in $3 \%$ of MM patients (1). In living patients, only one retrospective cohort study $(n=165)$ addressed this issue. Distant metastases were reported in up to $27 \%$ of all MM patients, depending on the affected organ site (2). Desmoplastic MM tends to metastasize more frequently than non-desmoplastic MM (3).

Neurological complaints in MM are common and may be attributed to peripheral causes such as peroneal nerve palsy (secondary to weight loss) or to treatment-related side effects (amongst others, polyneuropathy secondary to chemotherapeutics and immunotherapy-related toxicity). However, neurological symptoms due to metastasis to the central nervous system in a desmoplastic mesothelioma have never been reported before to our knowledge.

We present a case report of a desmoplastic MM patient with right leg paresis caused by a spinal cord metastasis.

\section{Clinical practice points}

- The differential diagnosis of neurological complaints in patients with malignant mesothelioma is broad.

- Hematogenous spinal cord metastasis of malignant mesothelioma is rare and has not been reported before in desmoplastic malignant mesothelioma.

- This case presents a desmoplastic malignant mesothelioma patient with right leg paresis caused by a spinal cord metastasis.

- This case demonstrates that spinal cord metastasis should be considered as a cause of neurological complaints in patients with malignant mesothelioma, especially when clinical examination suggests spinal cord pathology.

\section{Case report}

A 66-year-old male patient was diagnosed with a MM localized in the apical part of his right pleural cavity and invading the brachial plexus. Three months before, he underwent a foraminotomy at level C4-C7 for pain in his right neck and shoulder, without any improvement afterwards. After his MM was considered inoperable, he was treated with the combination of carboplatin (AUC5), pemetrexed $500 \mathrm{mg} / \mathrm{m}^{2}$, bevacizumab $15 \mathrm{mg} / \mathrm{kg}$ and atezolizumab $1200 \mathrm{mg}$ (after inclusion in the Multicenter Randomized Phase III 'BEAT-meso' trial of the European Thoracic Oncology Platform) (4). Under this treatment, he achieved a best response of 'stable disease'.

Five months after MM diagnosis, he was hospitalized because of paresis of the right leg with difficulties maintaining his equilibrium. Clinically, he had Brown-Séquard syndrome: pyramidal signs in the right leg (reduced muscle power, positive Babinski sign, slightly higher muscle tone and ankle clonus) and thermoanalgesia in the left hemicorpus, approximately up to level T3. On electrodiagnostic studies, a severe polyneuropathy was discovered, explaining why his deep sensation was severely diminished in both lower extremities.

Imaging of the spinal cord did not only reveal local spread of the MM at level T1-T2, but also a rightsided spinal metastasis at level C7-T1, compatible with the clinical findings (Figure 1,2). After 
multidisciplinary discussion, the BEAT trial treatment was stopped and locoregional radiotherapy on the primary MM tumor (25Gy) and the spinal metastasis (20Gy) was initiated, in combination with systemic steroids (methylprednisolone $16 \mathrm{mg}$ /day for 1 week and methylprednisolone $8 \mathrm{mg} / \mathrm{day}$ for 1 week) as an adjunctive anti-inflammatory treatment during the course of radiotherapy. There was no significant clinical improvement after this therapy and repeat CT one month later showed stable disease. In the months thereafter, his neurological status deteriorated and he received best supportive care.

\section{$\underline{\text { Discussion }}$}

Some case series already described the rare formation of intramedullary metastases in MM; however, none of these cases was of the histological desmoplastic subtype $(5,6)$. The mechanism of MM dissemination to the spinal cord remains intriguing but unprovable with imaging in our case. In figure $3 a$, we visualize a blood vessel with pathological widening and contrast captation, which originates in the lower intramedullary foramen. Consequently, hematogenous spread from the expanding primary MM tumor to this metastasis may have occurred via this route. In addition, local spread from the MM is visualized in figure $3 b$, extending into the pleura and soft tissue of the lung, further expanding in the right intervertebral foramen at level T1-T2, but not expanding into the extramedullar intraspinal space. Possibly the foraminotomy in our patient's history, with widening of the intervertebral spaces C4-C7, contributed to the local spread. Both hematogenous and local spread have been proposed as mechanisms of action in similar cases (5-7).

\section{References}

1. Finn R, Brims F, Gandhi A, Olsen N, Musk A, Maskell N et al. Postmortem findings of malignant pleural mesothelioma: a two-center study of 318 patients. Chest 2012;142(5):12671273.

2. Collins DC, Constantinidou A, Sundar R, Chenard-Poirier M, Yap TA, Banerji U et al. Patterns of metastases in malignant pleural mesothelioma in the modern era: Redefining the spread of an old disease. J Clin Oncol 2017;35(15):8556.

3. Cantin R, Jabi MA, McCaughey WT. Desmoplastic diffuse mesothelioma. Am J Surg Pathol 1982;6(3):215-222.

4. BEAT-meso: Bevacizumab and Atezolizumab in Malignant Pleural Mesothelioma (NCT03762018). Available from:

https://clinicaltrials.gov/ct2/show/NCT03762018?term=BEAT\&cond=Mesotheliomas+Pleural $\&$ draw $=2 \&$ rank $=1$ (accessed on June 01, 2020).

5. Yamamoto J, Ueta K, Takenaka M, Takahashi M, Nishizawa S. Sarcomatoid malignant mesothelioma presenting with intramedullary spinal cord metastasis: A case report and literature review. Global Spine J 2014;4(2):115-120.

6. Kalfas F, Skudieri C. Intramedullary Metastasis to the Cervical Spinal Cord from Malignant Pleural Mesothelioma: Review of the Literature and Case Reports. Asian J Neurosurg 2020;15(1):70-75.

7. Hillard VH, Liu JK, Kwok A, Schmidt MH. Perineural Spread of Malignant Mesothelioma Resulting in an Intradural Spinal Cord Mass: Case Report. J Neurooncol 2007; 81(2):185-89. 


\section{Figure legends}

Figure 1: Right paramedian sagittal T1-weighted image, showing the intramedullary mass lesion (larger white arrow) and posterior epidural lesion (smaller white arrow).

Figure 2: Median sagittal STIR image of the intramedullary mass lesion with surrounding edema.

Figure 3: Figure 3a (upper figure): Axial T1-weighted image after contrast with visualization of a pathologically thickened blood vessel against the tumor (white arrow). Figure $3 b$ (lower figure): Axial T1-weighted image after contrast, showing the extension of the tumor from the apex of the right lung to the intervertebral foramen (between arrows). 


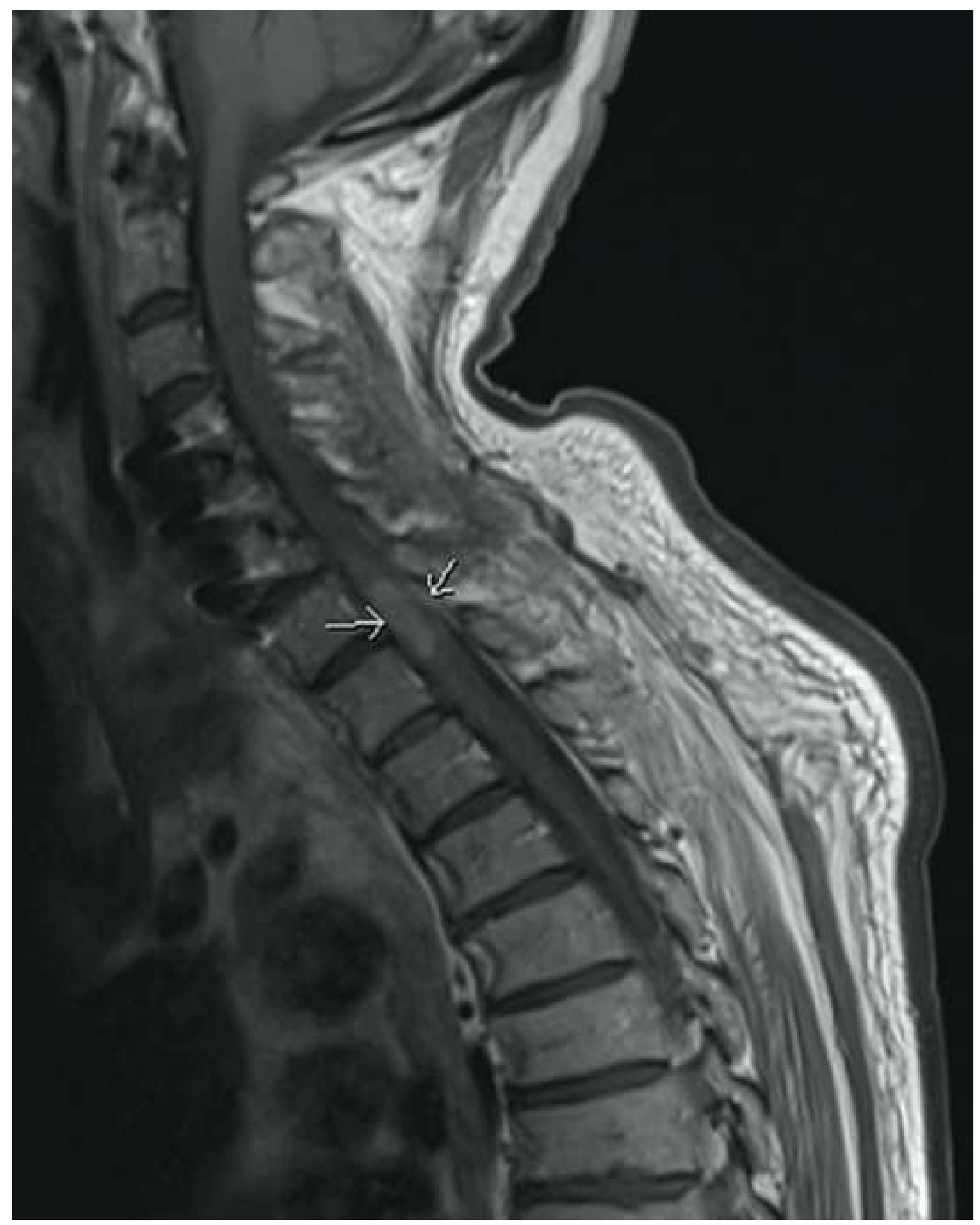




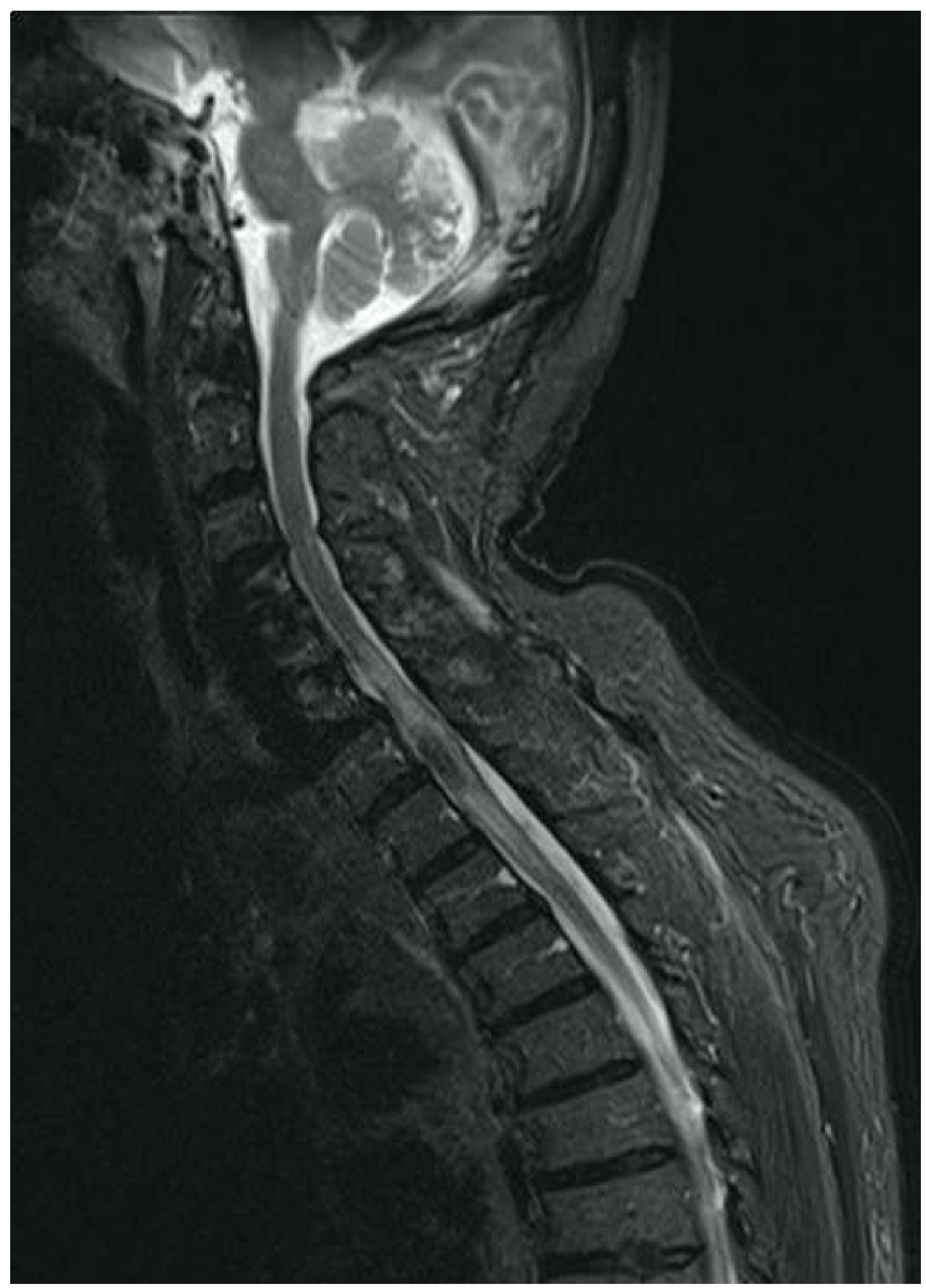



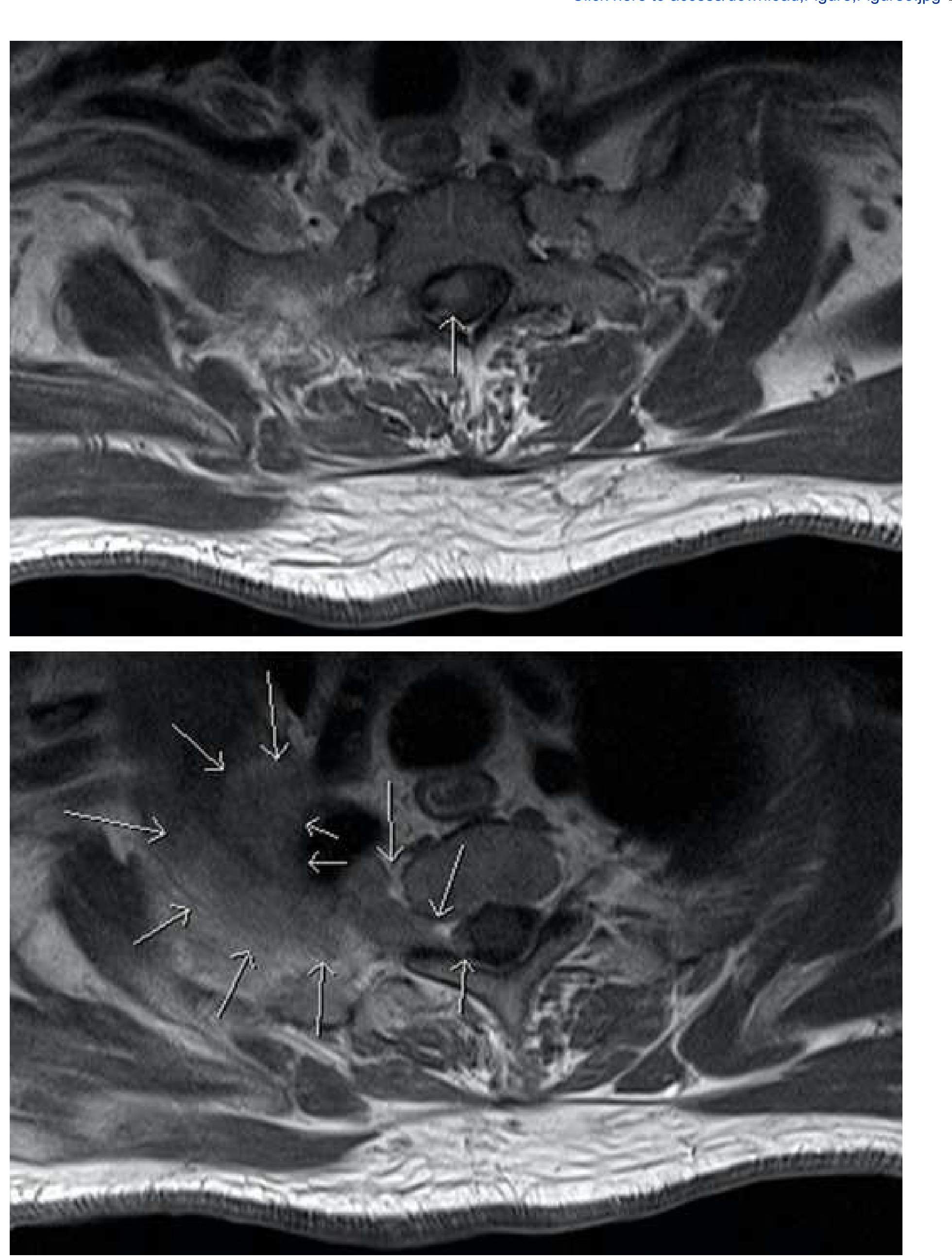

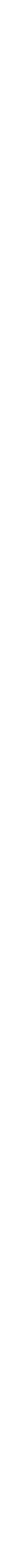

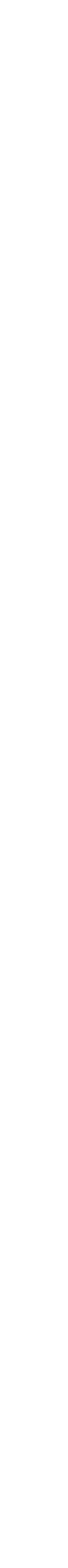




\section{Clinical practice points}

- The differential diagnosis of neurological complaints in patients with malignant mesothelioma is broad.

- Hematogenous spinal cord metastasis of malignant mesothelioma is rare and has not been reported before in desmoplastic malignant mesothelioma.

- This case presents a desmoplastic malignant mesothelioma patient with right leg paresis caused by a spinal cord metastasis.

- This case demonstrates that spinal cord metastasis should be considered as a cause of neurological complaints in patients with malignant mesothelioma, especially when clinical examination suggests spinal cord pathology. 